\title{
Lipopolysaccharides in Cyanobacteria: A Brief Overview
}

\author{
Sabrina Gemma, Monica Molteni, Carlo Rossetti \\ Dipartimento di Biotecnologie e Scienze della Vita, Università degli Studi dell'Insubria, Varese, Italy \\ Email: carlo.rossetti@uninsubria.it
}

Received 24 February 2016; accepted 26 April 2016; published 29 April 2016

Copyright (C) 2016 by authors and Scientific Research Publishing Inc.

This work is licensed under the Creative Commons Attribution International License (CC BY). http://creativecommons.org/licenses/by/4.0/

c) (i) Open Access

\begin{abstract}
Cyanobacteria are an interesting group of photosynthetic prokaryotes with a great potential in drug discovery and scientific research. Due to their high degree of diversification, they have been able to adapt to almost all ecological niches. Similarly to Gram-negative bacteria, cyanobacterial cell wall contains Lipopolysaccharides (LPSs) in the outer membrane layer. LPSs are molecules that possess the ability to elicit an innate immune response via Toll-like receptor 4 (TLR-4) activation. Cyanobacterial LPSs have been studied to a minor extent compared to Gram-negative bacterial LPSs. However, available data revealed important differences between the LPSs of these two groups of organisms, both in term of structure and biological activity. This review summarizes the current knowledge about cyanobacterial LPSs, highlighting their peculiarity and their potentiality compared to more characterized bacterial LPSs.
\end{abstract}

\section{Keywords}

Cyanobacteria, Lipopolysaccharide (LPS), Lipid A

\section{Introduction}

Cyanobacteria are among the oldest and most successful living organisms on earth. They are a highly diversified group of photosynthetic prokaryotes and can adapt to almost all ecological niches. They can be found in terrestrial, aerial, glaciers and aquatic environments, including extreme habitats like saline, hot springs and deserts [1]-[3]. Cyanobacteria are a huge source of bioactive compounds that can find applications in different markets including food, nutraceutical, pharmaceutical and cosmetic ones. Several cyanobacterial molecules have shown to possess pharmacologically interesting activities, like antiviral, antibacterial, antifungal, antimalarial, antitumor, immunosuppressant and many others [1]. Hence, cyanobacteria represent a great opportunity for drug discovery and scientific research. On the other hand, cyanobacterial metabolites include also toxins potentially 
harmful for animals and humans such as hepatotoxins, neurotoxins and endotoxins [4] [5].

Endotoxins, also known as lipopolysaccharides, are cell wall structures typical of Gram-negative bacteria that exhibit immunostimulatory activity [6] [7]. Cyanobacterial cell wall presents an overall resemblance with those of Gram-negative bacteria, including the presence of LPSs in the outer membrane layer [8]. LPS is made of a polysaccharidic portion and a lipidic portion that anchors the molecule to the outer membrane layer of cell wall [9]-[11]. LPSs are essential for the survival of bacteria because they contribute to membrane stability and integrity and constitute a first line defense system [6] [12].

The purpose of this review is to summarize the available knowledge about the structure and biological activity of cyanobacterial LPSs, highlighting their peculiarity and their potentiality compared to more characterized bacterial LPSs.

\section{LPSs in Gram-Negative Bacteria}

LPSs from Gram-negative bacteria have been extensively studied and LPSs from many different bacteria have been characterized. LPS consists of three different parts: a glycolipid portion called Lipid A; an oligosaccharidic core and a polysaccharidic chain, called O-antigen or O-specific chain [9]-[11] (Figure 1).

Lipid A is a glycophospholipidic structure that anchors the LPS molecule to the cellular membrane. This is a highly conserved portion with a $\beta$-1,6-diglucosamine (DGlcN) disaccharide backbone carrying phosphate groups at position C1 and C4', amide-linked fatty acid chains at position C3 and C3' and ester-linked fatty acid chains at position C2 and C2'. Hydroxylated fatty acid chains are frequently present (typically at C3 position) resulting in secondary acylation. The most typical acylation pattern is with $4-6$ chains ranging from 10 to 16 carbon atoms in length. Lipid A is linked to the proximal moiety of the oligosaccharidic core at position C6', where it binds covalently a 3-deoxy-D-manno-2-octulopyranosic acid (Kdo) [9]-[11].

The oligosaccharidic core consists of 10 - 12 sugars divided in a proximal and a distal region. The proximal region, called "inner core", contains Kdo, heptoses and negative charges usually derived from phosphate groups. The inner core has a remarkable importance for outer membrane integrity [6] [9] [10]. The distal region, called "outer core", consists usually of hexoses and shows more structural variability. This portion provides an attachment site for the O-antigen, if present [6] [9] [10].

The O-antigen is a polysaccharide made of repeated units. This portion is present only in smooth-type Gram-negative bacteria and shows a high heterogeneity in terms of length, composition and branching degrees. The role of this portion is variable in different bacteria. The O-antigen is known to modulate virulence, but evidences indicate that in some cases it may help bacterial evasion of host immune responses, in particular the complement cascade [6] [9] [10].

LPSs are known to elicit an innate immune response, triggering a cytokine cascade via TLR-4 activation [6] [7] [9] [13]. The immune response to LPS leads to a variety of symptoms ranging from fever to lethal sepsis [6] [11] [14]. Lipid A has been shown to be the biologically active moiety, but the polysaccharidic portions are known to also have an important role; indeed, Lipid A is usually less active than the entire LPS molecule

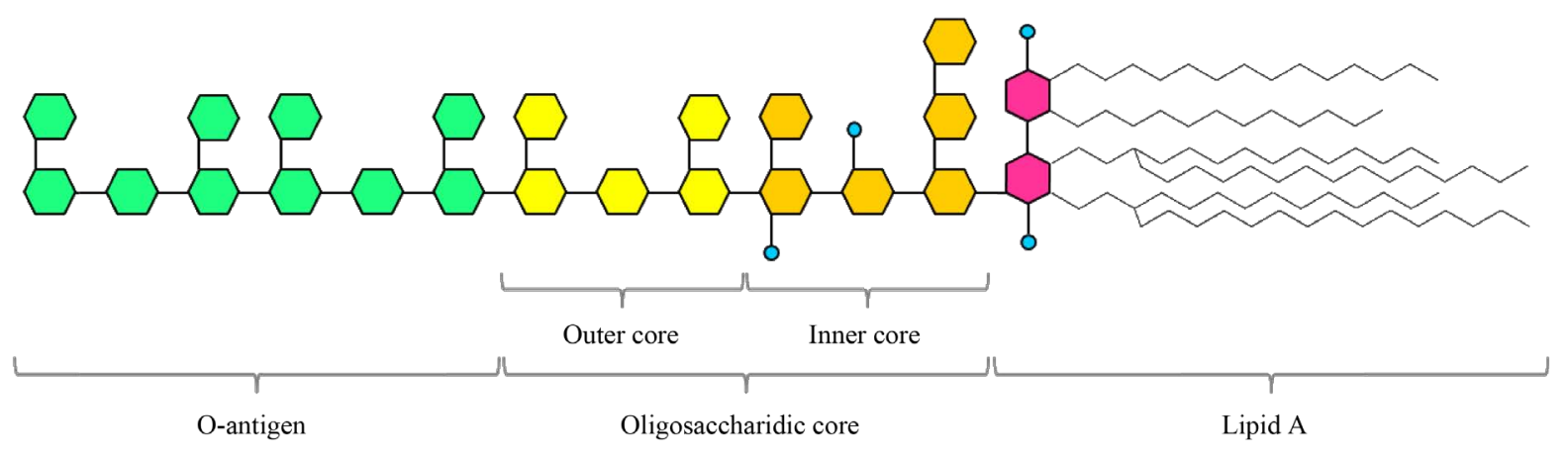

Monosaccharide $\circ$ Negative charge Fatty acid chain 
[9]-[11].

The highest endotoxic activity requires the presence in the Lipid A moiety of two phosphate groups on the disaccharide backbone and 6 acyl chains of 12 - 14 carbons of length, with two 3-acyloxyacyl groups. This is the so called "classic" structure of Lipid A (Figure 2). It should be noted that even small modifications of this pattern can affect dramatically the biological effect of LPS leading to a reduced effect or even, in some cases, to an antagonistic effect [9]-[11].

LPSs from cyanobacteria have been studied to a minor extent than those from Gram-negative bacteria both from structural and biological points of view [12]. Some recent studies tried to explore this field in order to better understand cyanobacterial LPSs.

\section{Structure of Cyanobacterial LPSs}

Between 70's and 80's a number of studies on cyanobacterial LPSs composition have been published, tough sometimes with contradictory results. In 1983 a study on Microcystis aeruginosa LPS suggested a structure rich in Kdo, 3-deoxy sugars, glucosamine, hexoses, phosphates and fatty acids while, some years later, another publication suggested a lack of Kdo and heptoses and the presence of several neutral sugars [15] [16]. This result has been recently confirmed showing a high prevalence of neutral sugars and no Kdo or heptose [17]. Several studies on different cyanobacterial species indicate the absence of heptose, Kdo and phosphate in cyanobacterial LPSs structures, in contrast with classic Gram-negative LPSs structures [16] [18]-[20]. Neutral sugars such as glucose, rhamnose, mannose and galactose are always present, while peculiar sugars are occasionally detected such as xylose, fucose and inositol [18]-[21]. Fatty acid composition was reported to be highly heterogeneous both in terms of length and degree of saturation, ranging from lauric acid (C12) to stearic acid (C18) with detection of polyunsaturated fatty acids like linoleic and linolenic acid [16] [18]-[22]. Long chain fatty acids and polyunsaturated fatty acids are not present in classic LPS structure derived from Gram-negative bacteria.

In 2009 the first structure of a cyanobacterial LPS was published. They studied two different Synechococcus strains (WH8102 and CC9311), which showed a high similarity in structure. Both strains showed a lack of Kdo, heptose and phosphate in their minimal structure, in agreement with previous studies. The core consisted of 6 glucoses, with the exception of a single rhamnose in WH8102 strain. Lipid A is a glucosamine disaccharide backbone linked to a galacturonic acid that replaces the phosphate group. As usual acylation pattern was heterogeneous, showing a predominance of tri- and tetra-acylated forms, with presence of both 3-hydroxilated and nonhydroxilated fatty acids, including long-chain fatty acids. The O-chain portion seemed to be a glucan-like

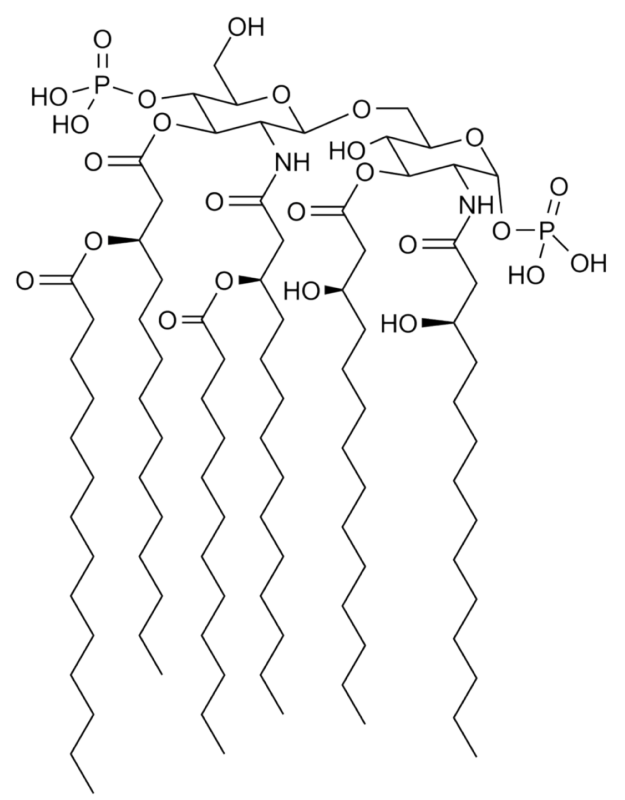

Figure 2. Classic highly endotoxic Lipid A structure from Escherichia coli (Source: Wikimedia Commons under CCZero license). 
polymer, due to the high abundance (near 100\%) of glucose in the complete LPS structure [12].

More recently an LPS structure from Oscillatoria planktothrix FP1 has been elucidated. The proposed structure showed a lack of heptose, Kdo and phosphate but a notable presence of uronic acid. The analysis performed suggested a rhamnan-like structure for the O-antigen. Rhamnose is the most abundant sugar in this LPS (more than $75 \%$ ) and the $\mathrm{O}$-antigen structure proposed is a long chain of $\alpha-1,3-\mathrm{Rhamnose}$ with irregular branches of different lengths and occasional glycosylation in position C2 or C4 of the rhamnose sugar. The oligosaccharidic core resulted to be rich in galacturonic acid, both in alfa and beta configuration, giving a strong negative charge to the region. In the Lipid A portion the presence of an acylated disaccharide glucosamine backbone linked to a galacturonic acid was confirmed. Acylation pattern showed the presence of at least two long chain (C16) 3-hydroxylated fatty acids [2].

Overall these studies highlighted important differences between the chemical structure of cyanobacterial and Gram-negative bacterial LPSs. By increasing the number of available LPSs structure derived from cyanobacteria, it will be possible to define a consensus structure for cyanobacterial LPS, similarly to what has been done for Gram-negative bacteria.

\section{Biological Activity of Cyanobacterial LPSs}

During the past decades several works have tried to evaluate the biological activity of cyanobacterial LPSs. Many cyanobacterial LPSs showed no toxicity [18]-[20] [23]-[25] or very low toxicity [15] [20] [22] [25] in comparison with classic Gram-negative LPSs when injected in mice. Despite these results some LPSs gave positive results in LAL test or in local Schwartzman reaction [15] [19]-[22] [25]-[27]. Overall these studies suggested that cyanobacterial LPSs are less endotoxic than classic Gram-negative LPSs. More detailed works appeared in the last decade.

In 2006 the first study to assess the biological activity of an LPS obtained from Oscillatoria planktothrix FP1 called CyP was published. This study showed that CyP could antagonize the inflammatory response elicited by E. coli LPS in human and mouse dendritic cells in a highly selective way, inhibiting both MyD88-dependent and MyD88-independent TLR-4 signaling pathways. Moreover this study showed that CyP was completely nontoxic both in human and mouse cells, and per se it did not induce an inflammatory response. CyP was also tested in vivo where it successfully protected mice from Salmonella abortus-equi LPS toxicity, reducing symptoms and overall mortality [28]. In a human whole blood model CyP was able to inhibit cytokine production induced not only through purified Neisseria meningitidis LPS, but also when cells were challenged by outer membrane vescicles or even live bacterial cells [29]. These results were confirmed later in a meningioma cell line and in an in vitro meningitis model infected with $N$. meningitidis [30]. Similarly, a porcine whole blood model showed that CyP suppressed cytokine expression induced by E. coli LPS. The same study showed that CyP could only moderately activate complement, suggesting a scarce ability to activate immune system in this animal model [31]. CyP ability to inhibit TLR-4 signaling resulted in an anticonvulsant effect in kainic acid-induced seizure, similarly to $R$. sphaeroides LPS, a commercial and well known TLR-4 antagonist [32]. De Paola et al. tested a more purified form of CyP (called VB3323) with in vitro and in vivo motor neuron degeneration models. They showed that TLR-4 antagonist activity of VB3323, similarly to R. sphaeorides LPS, resulted in a neuroprotective effect [33]. Thus, the activity of CyP was explored in a series of studies both in vitro and in vivo with consistent results showing a potent anti-inflammatory non species-specific activity. To our knowledge this is the most extensively characterized cyanobacterial LPS so far.

In 2011 Mayer et al. compared the effects of stimulation of rat microglia with Microcistis aeruginosa LPS in comparison with classic E. coli LPS. Firstly, they reported that higher doses (nearly 1000 fold) of M. aeruginosa LPS were necessary to induce the same toxic effect obtained with E. coli LPS. They also investigated a panel of markers to determine which LPS was more potent and more effective in inducing pro-inflammatory response. M. aeruginosa LPS resulted less potent than E. coli LPS in inducing pro-inflammatory mediators release, indeed higher doses of $M$. aeruginosa LPS were necessary to achieve maximum effect. However, M. aeruginosa LPS was more effective than $E$. coli LPS because when maximum effect was reached higher quantity of pro-inflammatory mediators were released, compared to E. coli LPS [5].

In 2016 Mayer et al. published an analogue study using an Oscillatoria sp. LPS in comparison with E. coli LPS. The experiments were performed on rat microglia using a wider panel of markers. Again, they showed that higher doses of Oscillatoria sp. LPS were necessary to induce the same toxic effect obtained with E. coli LPS. 
Overall Oscillatoria sp. LPS was less potent than E. coli LPS, but while its effectiveness was lower on nearly all of mediators tested, some exceptions were observed. For instance Oscillatoria sp. LPS induced 2.5 fold higher quantity of CXCL-1 chemokine and similar quantity of RANTES compared to E. coli LPS [34].

While a chemical structure has been proposed for the LPS extracted from Oscillatoria planktothrix FP1, there are no available data for the chemical structure or chemical composition of cyanobacterial LPSs evaluated in the two studies from Mayer et al. However, as a whole these studies confirm that cyanobacterial LPSs are able to stimulate immune response in different ways than classic and highly endotoxic LPSs. Further studies correlating biological activities and chemical structures of several cyanobacterial LPSs will be necessary to increase available knowledge and achieve a better understanding of the characteristic features of these molecules.

\section{Conclusions}

Cyanobacteria are an interesting source of new bioactive compounds but we have a scarce knowledge of these organisms. The presence of LPSs in cyanobacterial membrane gives them potential toxicity for humans and animals. However, our knowledge on this topic was based mainly on studies relative to Gram-negative bacterial LPSs, due to poor and often contradictory information on cyanobacterial LPSs.

Some recent studies regarding structure and function of cyanobacterial LPSs have filled this gap with interesting results. Several studies have demonstrated that cyanobacterial LPSs do not possess typical elements of classic and highly endotoxic Gram-negative LPS structures. Unsurprisingly, cyanobacterial LPSs tested on in vitro and in vivo models did not elicite strong immune responses. Interestingly, a fully characterized cyanobacterial LPS isolated from Oscillatoria planktothrix FP1 has shown to possess antagonistic effect on immune activation via TLR-4, resulting in a protective effect during infectious and noninfectious inflammatory processes.

In the next years, further research will be necessary to better understand this interesting group of microorganisms in order to fully exploit their huge potential.

\section{References}

[1] Dixit, R.B. and Suseela, M.R. (2013) Cyanobacteria: Potential Candidates for Drug Discovery. Antonie van Leeuwenhoek, 103, 947-961. http://dx.doi.org/10.1007/s10482-013-9898-0

[2] Carillo, S., Pieretti, G., Bedini, E., Parrilli, M., Lanzetta, R. and Corsaro, M.M. (2014) Structural Investigation of the Antagonist LPS from the Cyanobacterium Oscillatoria planktothrix FP1. Carbohydrate Research, 388, 73-80. http://dx.doi.org/10.1016/j.carres.2013.10.008

[3] Durai, P., Batool, M. and Choi, S. (2015) Structure and Effects of Cyanobacterial Lipopolysaccharides. Marine Drugs, 13, 4217-4230. http://dx.doi.org/10.3390/md13074217

[4] Giovannardi, S., Pollegioni, L., Pomati, F., Rossetti, C., Sacchi, S., Sessa, L., et al. (1999) Toxic Cyanobacterial Blooms in Lake Varese (Italy): A Multidisciplinary Approach. Environmental Toxicology, 14, 127-134. http://dx.doi.org/10.1002/(SICI)1522-7278(199902)14:1<127::AID-TOX16>3.0.CO;2-P

[5] Mayer, A.M.S., Clifford, J.A., Aldulescu, M., Frenkel, J.A., Holland, M.A., Hall, M.L., et al. (2011) Cyanobacterial Microcystis aeruginosa Lipopolysaccharide Elicits Release of Superoxide Anion, Thromboxane $\mathrm{B}_{2}$, Cytokines, Chemokines, and Matrix Metalloproteinase-9 by Rat Microglia. Toxicological Sciences, 121, 63-72. http://dx.doi.org/10.1093/toxsci/kfr045

[6] Raetz, C.R.H. and Whitfield, C. (2002) Lipopolysaccharide Endotoxins. Annual Review of Biochemistry, 71, 635-700. http://dx.doi.org/10.1146/annurev.biochem.71.110601.135414

[7] Beutler, B. and Rietschel, E.T. (2003) Innate Immune Sensing and Its Roots: The Story of Endotoxin. Nature Reviews, Immunology, 3, 169-176. http://dx.doi.org/10.1038/nri1004

[8] Hoiczyk, E. and Hansel, A. (2000) Cyanobacterial Cell Walls: News from an Unusual Prokaryotic Envelope. Journal of Bacteriology, 182, 1191-1199. http://dx.doi.org/10.1128/JB.182.5.1191-1199.2000

[9] Caroff, M., Karibian, D., Cavaillon, J.M. and Haeffner-Cavaillon, N. (2002) Structural and Functional Analyses of Bacterial Lipopolysaccharides. Microbes and Infection, 4, 915-926. http://dx.doi.org/10.1016/S1286-4579(02)01612-X

[10] Caroff, M. and Karibian, D. (2003) Structure of Bacterial Lipopolysaccharides. Carbohydrate Research, 338, 24312447. http://dx.doi.org/10.1016/j.carres.2003.07.010

[11] Dixon, D.R. and Darveau, R.P. (2005) Lipopolysaccharide Heterogeneity: Innate Host Responses to Bacterial Modification of Lipid A Structure. Journal of Dental Research, 84, 584-595. http://dx.doi.org/10.1177/154405910508400702

[12] Snyder, D.S., Brahamsha, B., Azadi, P. and Palenik, B. (2009) Structure of Compositionally Simple Lipopolysaccha- 
ride from Marine Synechococcus. Journal of Bacteriology, 191, 5499-5509. http://dx.doi.org/10.1128/JB.00121-09

[13] Akira, S. and Takeda, K. (2004) Toll-Like Receptor Signalling. Nature Reviews Immunology, 4, 499-511.

[14] Stewart, I., Schluter, P.J. and Shaw, G.R. (2006) Cyanobacterial Lipopolysaccharides and Human Health-A Review. Environmental Health, 5, 7. http://dx.doi.org/10.1186/1476-069X-5-7

[15] Raziuddin, S., Siegelman, H.W. and Tornabene, T.G. (1983) Lipopolysaccharides of the Cyanobacterium Microcystis aeruginosa. The FEBS Journal, 137, 333-336. http://dx.doi.org/10.1111/j.1432-1033.1983.tb07833.x

[16] Martin, C., Codd, G.A., Siegelman, H.W. and Weckesser, J. (1989) Lipopolysaccharides and Polysaccharides of the Cell Envelope of Toxic Microcystis aeruginosa Strains. Archives of Microbiology, 152, 90-94. http://dx.doi.org/10.1007/BF00447017

[17] Fujii, M., Sato, Y., Ito, H., Masago, Y. and Omura, T. (2012) Monosaccharide Composition of the Outer Membrane Lipopolysaccharide and O-Chain from the Freshwater Cyanobacterium Microcystis aeruginosa NIES-87. Journal of Applied Microbiology, 113, 896-903. http://dx.doi.org/10.1111/j.1365-2672.2012.05405.x

[18] Weise, G., Drews, G., Jann, B. and Jann, K. (1970) Identification and Analysis of a Lipopolysaccharide in Cell Walls of the Blue-Green Alga Anacystis nidulans. Archiv für Mikrobiologie, 71, 89-98.

[19] Keleti, G., Sykora, J.L., Lippy, E.C. and Shapiro, M.A. (1979) Composition and Biological Properties of Lipopolysaccharides Isolated from Schizothrix calcicola (Ag.) Gomont (Cyanobacteria). Applied and Environmental Microbiology, 38, 471-477.

[20] Keleti, G. and Sykora, J.L. (1982) Production and Properties of Cyanobacterial Endotoxins. Applied and Environmental Microbiology, 43, 104-109.

[21] Buttke, T.M. and Ingram, L.O. (1975) Comparison of Lipopolysaccharides from Agmenellum quadruplicatum to Escherichia coli and Salmonella typhimurium by Using Thin-Layer Chromatography. Journal of Bacteriology, 124, 1566-1573.

[22] Tornabene, T., Bourne, T., Raziuddin, S. and Ben-Amotz, A. (1985) Lipid and Lipopolysaccharide Constituents of Cyanobacterium Spirulina platensis (Cyanophyceae, Nostocales). Marine Ecology-Progress Series, 22, 121-125. http://dx.doi.org/10.3354/meps022121

[23] Katz, A., Weckesser, J., Drews, G. and Mayer, H. (1977) Chemical and Biological Studies on the Lipopolysaccharide (O-Antigen) of Anacystis nidulans. Archives of Microbiology, 113, 247-256. http://dx.doi.org/10.1007/BF00492032

[24] Mikheyskaya, L.V., Ovodova, R.G. and Ovodov, Y.S. (1977) Isolation and Characterization of Lipopolysaccharides from Cell Walls of Blue-Green Algae of the Genus Phormidium. Journal of Bacteriology, 130, 1-3.

[25] Mohamed, Z.A. (2008) Toxic Cyanobacteria and Cyanotoxins in Public Hot Springs in Saudi Arabia. Toxicon, 51, 17-27. http://dx.doi.org/10.1016/j.toxicon.2007.07.007

[26] Rapala, J. (2002) Endotoxins Associated with Cyanobacteria and Their Removal during Drinking Water Treatment. Water Research, 36, 2627-2635. http://dx.doi.org/10.1016/S0043-1354(01)00478-X

[27] Bernardová, K., Babica, P., Marsálek, B. and Bláha, L. (2008) Isolation and Endotoxin Activities of Lipopolysaccharides from Cyanobacterial Cultures and Complex Water Blooms and Comparison with the Effects of Heterotrophic Bacteria and Green Alga. Journal of Applied Toxicology, 28, 72-79. http://dx.doi.org/10.1002/jat.1257

[28] Macagno, A., Molteni, M., Rinaldi, A., Bertoni, F., Lanzavecchia, A., Rossetti, C., et al. (2006) A Cyanobacterial LPS Antagonist Prevents Endotoxin Shock and Blocks Sustained TLR4 Stimulation Required for Cytokine Expression. The Journal of Experimental Medicine, 203, 1481-1492. http://dx.doi.org/10.1084/jem.20060136

[29] Jemmett, K., Macagno, A., Molteni, M., Heckels, J.E., Rossetti, C. and Christodoulides, M. (2008) A Cyanobacterial Lipopolysaccharide Antagonist Inhibits Cytokine Production Induced by Neisseria meningitidis in a Human WholeBlood Model of Septicemia. Infection and Immunity, 76, 3156-3163. http://dx.doi.org/10.1128/IAI.00110-08

[30] Oliver, R., Staples, K.J., Heckels, J., Rossetti, C., Molteni, M. and Christodoulides, M. (2012) Coadministration of the Cyanobacterial Lipopolysaccharide Antagonist CyP with Antibiotic Inhibits Cytokine Production by an in Vitro Meningitis Model Infected with Neisseria meningitidis. Journal of Antimicrobial Chemotherapy, 67, 1145-1154. http://dx.doi.org/10.1093/jac/dks031

[31] Thorgersen, E.B., Macagno, A., Rossetti, C. and Mollnes, T.E. (2008) Cyanobacterial LPS Antagonist (CyP)—A Novel and Efficient Inhibitor of Escherichia coli LPS-Induced Cytokine Response in the Pig. Molecular Immunology, 45, 3553-3557. http://dx.doi.org/10.1016/j.molimm.2008.05.005

[32] Maroso, M., Balosso, S., Ravizza, T., Liu, J., Aronica, E., Iyer, A.M., et al. (2010) Toll-Like Receptor 4 and HighMobility Group Box-1 Are Involved in Ictogenesis and Can Be Targeted to Reduce Seizures. Nature Medicine, 16, 413-419. http://dx.doi.org/10.1038/nm.2127

[33] DePaola, M., Mariani, A., Bigini, P., Peviani, M., Ferrara, G., Molteni, M., et al. (2012) Neuroprotective Effects of Toll-Like Receptor 4 Antagonism in Spinal Cord Cultures and in a Mouse Model of Motor Neuron Degeneration. Mo- 
lecular Medicine, 18, 971-981.

[34] Mayer, A.M.S., Murphy, J., MacAdam, D., Osterbauer, C., Baseer, I., Hall, M.L., et al. (2016) Classical and Alternative Activation of Cyanobacterium Oscillatoria sp. Lipopolysaccharide-Treated Rat Microglia in Vitro. Toxicological Sciences, 149, 484-495. http://dx.doi.org/10.1093/toxsci/kfv251 